\title{
Transversely Isotropic Problems
}

\subsection{Introduction}

A transversely isotropic medium is a medium which has a favored direction and is isotropic in the plane perpendicular to this direction. Among crystalline media, all materials with a hexagonal crystal system belong to this class: they are elastically isotropic in the plane perpendicular to the hexagonal axis. Fiber composites with the fibers arranged in parallel in one direction also represent a transversely isotropic medium, which is isotropic in the plane perpendicular to the fiber direction (see Fig. 7.1). Many functional materials exhibiting a preferred direction can also be classified as such, e.g., some piezo-electric materials. We can find many more examples in biological media.

A linear transversely isotropic medium is fully defined by five elastic constants. For the definition of these constants using the elastic moduli and coefficients of transverse contraction, see Fig. 7.1. If we call the axis of symmetry of the medium " $z$ ", the axes " $x$ " and " $y$ " are "equivalent" and they can be defined arbitrarily in the plane spanned by these two axes.

Fig. 7.1 Demonstration of the symmetry and definition of elastic constants of a transversely isotropic medium

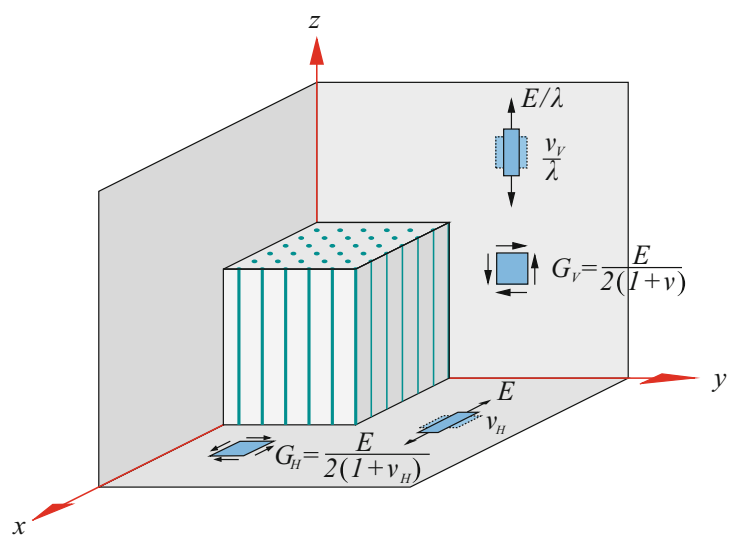


Turner (1980) described the relationship between the deformation tensor and the stress tensor using the matrix of compliance coefficients:

$$
\left[\begin{array}{c}
\varepsilon_{x x} \\
\varepsilon_{y y} \\
\varepsilon_{z z} \\
2 \varepsilon_{x z} \\
2 \varepsilon_{y z} \\
2 \varepsilon_{x y}
\end{array}\right]=\frac{1}{E}\left[\begin{array}{cccccc}
1 & -v_{H} & -v_{V} & 0 & 0 & 0 \\
-v_{H} & 1 & -v_{V} & 0 & 0 & 0 \\
-v_{V} & -v_{V} & \lambda & 0 & 0 & 0 \\
0 & 0 & 0 & 2(1+v) & 0 & 0 \\
0 & 0 & 0 & 0 & 2(1+v) & 0 \\
0 & 0 & 0 & 0 & 0 & 2\left(1+v_{H}\right)
\end{array}\right]\left[\begin{array}{c}
\sigma_{x x} \\
\sigma_{y y} \\
\sigma_{z z} \\
\sigma_{x z} \\
\sigma_{y z} \\
\sigma_{x y}
\end{array}\right],
$$

where

$$
\varepsilon_{i j}=\frac{1}{2}\left(\frac{\partial u_{i}}{\partial x_{j}}+\frac{\partial u_{j}}{\partial x_{i}}\right)
$$

is the linear symmetric deformation tensor and $u_{i}$ is the displacement vector. Here, $E$ is the elasticity modulus of the medium in the plane perpendicular to the axis of symmetry, $E / \lambda$ is the elasticity modulus in the direction of the axis of symmetry, $v_{H}$ is the Poisson's ratio in the plane perpendicular to the axis of symmetry, and $\nu_{V}$ is the Poisson's ratio when stress is applied along the symmetry axis. $G_{V}=$ $E /(2+2 v)$ is the shear modulus for shear parallel to the axis of symmetry; note that $v$ has no immediate physical meaning. Additionally, it should be noted that the shear modulus in the plane of symmetry is given by the usual equation $G_{H}=$ $E /\left(2+2 v_{H}\right)$.

Inverting the system of equations leads to the presentation via the matrix of stiffness coefficients:

$$
\left[\begin{array}{c}
\sigma_{x x} \\
\sigma_{y y} \\
\sigma_{z z} \\
\sigma_{x z} \\
\sigma_{y z} \\
\sigma_{x y}
\end{array}\right]=\left[\begin{array}{cccccc}
C_{11} & C_{12} & C_{13} & 0 & 0 & 0 \\
C_{12} & C_{11} & C_{13} & 0 & 0 & 0 \\
C_{13} & C_{13} & C_{33} & 0 & 0 & 0 \\
0 & 0 & 0 & C_{44} & 0 & 0 \\
0 & 0 & 0 & 0 & C_{44} & 0 \\
0 & 0 & 0 & 0 & 0 & \frac{1}{2}\left(C_{11}-C_{12}\right)
\end{array}\right]\left[\begin{array}{c}
\varepsilon_{x x} \\
\varepsilon_{y y} \\
\varepsilon_{z z} \\
2 \varepsilon_{x z} \\
2 \varepsilon_{y z} \\
2 \varepsilon_{x y}
\end{array}\right]
$$

The elastic constants can be written in Voigt notation, as follows:

$$
\begin{aligned}
C_{11} & =\frac{E\left(\lambda-v_{V}^{2}\right)}{\left(\lambda-\lambda v_{H}-2 v_{V}^{2}\right)\left(1+v_{H}\right)}, \\
C_{12} & =\frac{E\left(\lambda v_{H}+v_{V}^{2}\right)}{\left(\lambda-\lambda v_{H}-2 v_{V}^{2}\right)\left(1+v_{H}\right)}, \\
C_{13} & =\frac{v_{V} E}{\left(\lambda-\lambda v_{H}-2 v_{V}^{2}\right)},
\end{aligned}
$$




$$
\begin{aligned}
C_{33} & =\frac{\left(1-v_{H}\right) E}{\left(\lambda-\lambda v_{H}-2 v_{V}^{2}\right)}, \\
C_{44} & =\frac{E}{2(1+v)}, \\
\frac{1}{2}\left(C_{11}-C_{12}\right) & =\frac{E}{2\left(1+v_{H}\right)},
\end{aligned}
$$

using the moduli and Poisson's ratios.

\subsection{Normal Contact Without Adhesion}

For the complete formulation of the contact mechanical problem in its integral form, it is sufficient to know the fundamental solution, independent of the class of symmetry of the medium. The fundamental solution for transversely isotropic media was found by Michell (1900). He demonstrated that the normal displacement $w$ of the surface of a transversely isotropic elastic half-space under the effect of a force $F_{z}$ acting on the origin is given by the equation:

$$
w(r)=\frac{1}{\pi E^{*}} \frac{F_{z}}{r},
$$

where $r$ is the distance in-plane to the acting point of the force. The equation has the same form as the corresponding fundamental solution for the case of isotropic media, as shown in (2.2). It simply requires the following definition of the effective elasticity modulus:

$$
E^{*}=\frac{2 \sqrt{C_{44}}\left(C_{11} C_{33}-C_{13}^{2}\right)}{\sqrt{C_{11}} \sqrt{\left(\sqrt{C_{11} C_{33}}-C_{13}\right)\left(C_{13}+2 C_{44}+\sqrt{C_{11} C_{33}}\right)}} .
$$

As such, Michell (1900) concluded that:

It appears, therefore, that the law of depression is the same as for an isotropic solid; consequently, the applications of this law, which were made by Boussinesq and Hertz to problems concerning isotropic bodies in contact, may be at once extended to the acolotropic solids here considered, with the limitation that the normal to the plane of contact must be an axis of elastic symmetry.

Of course, the effective elasticity modulus can also be expressed by the components of the compliance matrix:

$$
E^{*}=\frac{E}{\left(1-v_{H}^{2}\right)} \cdot \sqrt{\frac{2}{\left(\frac{\lambda-v_{V}^{2}}{1-v_{H}^{2}}\right)^{1 / 2}+\frac{1+v-v_{V}\left(1+v_{H}\right)}{1-v_{H}^{2}}}} .
$$


In the case of an isotropic continuum $\left(\lambda=1, v_{V}=v_{H}=v\right)$, this expression is reduced to the known equation $E^{*}=E /\left(1-v^{2}\right)$.

The integral formulation of (2.3) for the frictionless contact mechanical problem is based exclusively on the fundamental solution. Therefore, all solutions from Chap. 2 are equally valid for transversely isotropic media.

When two transversely isotropic bodies are in contact, the effective modulus is used instead of (7.6):

$$
\frac{1}{E^{*}}=\frac{1}{E_{1}^{*}}+\frac{1}{E_{2}^{*}},
$$

where $E_{1}^{*}$ and $E_{2}^{*}$ represent the effective elastic moduli of the two media.

Likewise, due to the identical fundamental solutions, applying the MDR to a transversely isotropic medium simply requires substituting the effective elasticity modulus by the expressions presented in (7.6) and (7.8). All other transformation rules of the MDR remain unchanged.

Therefore, the non-adhesive normal contact problem for a transversely isotropic medium is identical to the corresponding contact problem of an isotropic continuum. This applies to the displacement field of the surface of the body and the pressure distribution in the immediate surface, but not to the deformation and stress distribution in the interior of the half-space. Consequently, there is no need for special consideration to be given to all normal contact problems for transversely isotropic media. We will simply refer to the results from Chap. 2, which are equally valid for transversely isotropic media.

Further information, particularly concerning the calculation of the stresses in the interior of the transversely isotropic half-space (which, again, do not coincide with those of the isotropic case), can be found in a paper by $\mathrm{Yu}$ (2001).

For a historical perspective, the paper by Conway (1956) is worth mentioning. Notably, it describes how, due to the form of the fundamental solution (7.5) by Michell (1900), the calculation method for any (isotropic) axially symmetric normal contact problem by Schubert (1942) can also be applied to the corresponding contact problem of transversely isotropic media.

\subsection{Normal Contact with Adhesion}

As explained in the previous section, the non-adhesive, frictionless normal contact problem for a transversely isotropic medium is identical to the corresponding contact problem for an isotropic continuum. It merely requires redefining the effective elasticity modulus according to (7.6) or (7.7). Additionally, in Chap. 3 of this book, it was shown that the adhesive, frictionless normal contact problem can be reduced to the corresponding non-adhesive contact. Therefore, the adhesive normal contact problems of isotropic and transversely isotropic media are also equivalent to the respective isotropic problems, regarding both their relationships of the global contact quantities (normal force, indentation depth, and contact radius), as well as the stresses in the contact surface, and the displacements of the medium's surface. 
Consequently, there is no need for special consideration to be given to adhesive normal contact problems of transversely isotropic media. Here we will simply refer to the results in Chap. 3 of this book. Taking into account the aforementioned corresponding definition of the effective elasticity modulus, the results directly apply to transversely isotropic contacts.

An overview of the history of work done in the field of adhesive contacts of transversely isotropic media can be found in an article by Borodich et al. (2014).

\subsection{Tangential Contact}

Turner (1980) provided a general expression for the surface displacement of a transversely isotropic elastic half-space under the effect of an arbitrarily directed force acting on the surface of the half-space at the origin. He used the matrix of compliance coefficients, as seen in (7.1).

According to Turner, the simultaneous effect of a normal force $F_{N}$ and a tangential force $F_{x}$ in the $x$-direction generates the surface displacements

$$
\left[\begin{array}{l}
u(x, y) \\
v(x, y) \\
w(x, y)
\end{array}\right]=\frac{\varepsilon}{2 \pi r}\left[\begin{array}{l}
\gamma(x / r) F_{N}+\left(1+\delta(x / r)^{2}\right) F_{x} \\
\gamma(y / r) F_{N}+\delta\left(x y / r^{2}\right) F_{x} \\
\left.\alpha F_{N}-\gamma(x / r)\right) F_{x}
\end{array}\right], \quad r^{2}=x^{2}+y^{2},
$$

with

$$
\begin{aligned}
\alpha & =\left(\frac{\lambda-v_{V}^{2}}{1-v_{H}^{2}}\right)^{1 / 2}, \\
\beta & =\frac{(1+v)-v_{V}\left(1+v_{H}\right)}{\left(1-v_{H}^{2}\right)}, \\
\gamma & =\left(\frac{2}{\alpha+\beta}\right)^{1 / 2}\left(\frac{\alpha}{2}-\frac{v_{V}}{2\left(1-v_{H}\right)}\right), \\
\delta & =\left(\frac{2}{\alpha+\beta}\right)^{1 / 2}\left(\frac{1+v}{1+v_{H}}\right)^{1 / 2} \frac{1}{1-v_{H}}-1, \\
\varepsilon & =\left(\frac{\alpha+\beta}{2}\right)^{1 / 2} \frac{1-v_{H}}{G_{H}} .
\end{aligned}
$$

For an isotropic medium, $\lambda=1, \alpha=\beta=1, \gamma=(1-2 v) /(2-2 v), \delta=v /(1-v)$, and $\varepsilon=(1-v) / G ;(7.9)$ can then be reduced to the form provided by Landau and Lifshitz (1944, 1959):

$$
\begin{aligned}
& u(x, y)=\frac{1+v}{2 \pi E} \frac{1}{r}\left\{-(1-2 v) \frac{x}{r} F_{N}+\left(2(1-v)+\frac{2 v x^{2}}{r^{2}}\right) F_{x}\right\}, \\
& v(x, y)=\frac{1+v}{2 \pi E} \frac{1}{r}\left\{-(1-2 v) \frac{y}{r} F_{N}+2 v \frac{x y}{r^{2}} F_{x}\right\}, \quad r^{2}=x^{2}+y^{2}, \\
& w(x, y)=\frac{1+v}{2 \pi E} \frac{1}{r}\left\{2(1-v) F_{N}+(1-2 v) \frac{x}{r} F_{x}\right\} .
\end{aligned}
$$


The normal and tangential contact problems are independent of one another for a vanishing $\gamma$ in (7.9), i.e., when

$$
\frac{1-v_{H}}{1+v_{H}} \frac{\lambda-v_{V}^{2}}{v_{V}^{2}}=1 .
$$

The criterion for the decoupling of the normal and tangential contact problem in the case of an isotropic continuum is reduced to the requirement of incompressibility $(v=1 / 2)$ of the deformable contact partner. In the case that both media are linearelastic and transversely isotropic, the quantity $\gamma_{1}-\gamma_{2}$ must vanish:

$$
\begin{aligned}
& \left(\frac{2}{\alpha_{1}+\beta_{1}}\right)^{1 / 2}\left(\frac{\alpha_{1}}{2}-\frac{v_{V, 1}}{2\left(1-v_{H, 1}\right)}\right) \\
& -\left(\frac{2}{\alpha_{2}+\beta_{2}}\right)^{1 / 2}\left(\frac{\alpha_{2}}{2}-\frac{v_{V, 2}}{2\left(1-v_{H, 2}\right)}\right)=0 .
\end{aligned}
$$

\subsection{1 "Cattaneo-Mindlin" Approximation for the Transversely Isotropic Contact}

Assuming a decoupling of the normal and tangential contact problem $(\gamma=0)$, and neglecting the surface displacement in the direction perpendicular to the direction of force action (as assumed by the solution by Cattaneo and Mindlin in Chap. 4), (7.9) and (7.11) can be simplified to:

$$
\left[\begin{array}{c}
u(r) \\
w(r)
\end{array}\right]=\frac{\varepsilon}{2 \pi r}\left[\begin{array}{c}
\left(1+\delta(x / r)^{2}\right) F_{x} \\
\alpha F_{N}
\end{array}\right],
$$

for a transversely isotropic medium and to:

$$
\left[\begin{array}{c}
u(r) \\
w(r)
\end{array}\right]=\frac{1+v}{2 \pi E} \frac{1}{r}\left[\begin{array}{c}
\left(2(1-v)+2 v(x / r)^{2}\right) F_{x} \\
2(1-v) F_{N}
\end{array}\right],
$$

for an isotropic medium. It is easy to see that the expression for the tangential displacements in a transversely isotropic medium exactly matches the one for an isotropic medium for the values $\varepsilon=2\left(1-v^{2}\right) / E$ and $\delta=v /(1-v)$.

Solving for $E$ and $v$ gives $v=\frac{\delta}{1+\delta}$ and $E=\frac{2\left(1-v^{2}\right)}{\varepsilon}=\frac{2(1+2 \delta)}{\varepsilon(1+\delta)^{2}}$. For the effective shear modulus, we obtain:

$$
G^{*}=\frac{4 G}{2-v}=\frac{2 E}{(1+v)(2-v)}=\frac{4}{\varepsilon(2+\delta)} .
$$

Similarly, achieving identical normal displacements for both transversely isotropic and isotropic media requires the effective moduli to follow the expressions

$$
E^{*}=\frac{2}{\alpha \varepsilon} .
$$


Inserting the definitions of $\delta, \alpha$, and $\varepsilon$ from (7.10) yields the following result:

$$
G^{*}=\frac{2 E}{\left(1+v_{H}\right)\left(\left(1-v_{H}\right)\left(\frac{1}{2}\left(\frac{1-v_{V}^{2}}{1-v_{H}^{2}}\right)^{1 / 2}+\frac{1}{2} \frac{1+v-v_{V}\left(1+v_{H}\right)}{1-v_{H}^{2}}\right)+\left(\frac{1+v}{1+v_{H}}\right)^{1 / 2}\right)},
$$

which, for the isotropic continuum $\left(\lambda=1, v_{V}=v_{H}=v\right)$, takes on the usual form of:

$$
G^{*}=\frac{2 E}{(1+v)(2-v)} .
$$

Inserting the definitions of $\alpha$ and $\varepsilon$ from (7.10) into (7.17) leads to the expression previously formulated in (7.7).

Taking this into consideration, this proves the equivalence of the fundamental solutions for the normal contact problem and the tangential contact problem in the Cattaneo-Mindlin approximation for isotropic and transversely isotropic continua. Therefore, using definitions (7.16), (7.17), (7.18), and (7.7) of the effective moduli, all results from Chaps. 2, 3, and 4 regarding the relationships of the macroscopical displacements, forces, contact radii, and stress distributions carry over. Only the stresses in the interior of the medium require special consideration.

The ratio of normal to tangential stiffness of a no-slip contact is given by:

$$
\frac{E^{*}}{G^{*}}=\frac{2+\delta}{2 \alpha}
$$

which (in the isotropic case) is reduced to the Mindlin ratio:

$$
\frac{E^{*}}{G^{*}}=\frac{2-v}{2-2 v}
$$

\subsection{Summary of the Calculation of Transversely Isotropic Contacts}

Once again, we will provide a summary of the approach to solving contact problems of transversely isotropic media.

The quantities defined at the surface of the medium are listed below:

- Normal force

- Contact radius

- Indentation depth

- Distribution of normal stresses and normal displacements at the surface

- The quantities previously listed for the adhesive contact in the JKR approximation

- Tangential force in contact with friction in the "Cattaneo-Mindlin approximation" 
- Macroscopical tangential displacement in contact with friction in the "CattaneoMindlin approximation"

- Distribution of tangential stresses and tangential displacements at the surface in the "Cattaneo-Mindlin approximation"

Transversely isotropic media exhibit exactly the same behavior as isotropic media. The only required change involves inserting the effective elasticity modulus (defined in (7.6) and equivalently in (7.7)) or the effective shear modulus defined in (7.18), respectively. The MDR method is also valid without restriction, unchanged from the case of isotropic media.

\section{References}

Borodich, F.M., Galanov, B.A., Keer, L.M., Suarez-Alvarez, M.M.: The JKR-type adhesive contact problems for transversely isotropic elastic solids. Mech. Mater. 75, 34-44 (2014)

Conway, H.D.: The indentation of a transversely isotropic half-space by a rigid punch. Z. Angew. Math. Phys. 7(1), 80-85 (1956)

Landau, L.D., Lifshitz, E.M.: Механика сплошных сред. Гидродинамика и теория упругости. Теоретическая физика, vol. III. ОГИЗ. ГИТТЛ, Москва (1944)

Landau, L.D., Lifshitz, E.M.: Theory of elasticity. Course of theoretical physics, vol. 7. Pergamon Press, London (1959)

Michell, J.H.: The stress in an aeolotropie elastic solid with an infinite plane boundary. Proc. London Math. Soc. 32, 247-258 (1900)

Schubert, G.: Zur Frage der Druckverteilung unter elastisch gelagerten Tragwerken. Ing. Arch. 13(3), 132-147 (1942)

Turner, J.R.: Contact on a transversely isotropic half-space, or between two transversely isotropic bodies. Int. J. Solids Struct. 16, 409-419 (1980)

Yu, H.Y.: A concise treatment of indentation problems in transversely isotropic half-spaces. Int. J. Solids Struct. 38(10), 2213-2232 (2001)

Open Access This chapter is licensed under the terms of the Creative Commons Attribution 4.0 International License (http://creativecommons.org/licenses/by/4.0/), which permits use, sharing, adaptation, distribution and reproduction in any medium or format, as long as you give appropriate credit to the original author(s) and the source, provide a link to the Creative Commons license and indicate if changes were made.

The images or other third party material in this chapter are included in the chapter's Creative Commons license, unless indicated otherwise in a credit line to the material. If material is not included in the chapter's Creative Commons license and your intended use is not permitted by statutory regulation or exceeds the permitted use, you will need to obtain permission directly from the copyright holder.

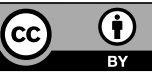

